\title{
Principais caracteres da osteologia craniana de Milvago chimango (Vieillot, 1816) e Milvago chimachima (Vieillot, 1816) (Aves: Falconidae)
}

\author{
Anderson Guzzi ${ }^{1}$ \\ Antonia Marques dos Santos ${ }^{1}$ \\ Juliana Dias dos Santos ${ }^{1}$ \\ Reginaldo José Donatelli ${ }^{2}$ \\ Guilherme José Bolzani de Campos Ferreira ${ }^{3 *}$ \\ ${ }^{1}$ Universidade Federal do Piauí, Curso de Ciências Biológicas, Departamento de Ciências do Mar Campus Ministro Reis Velloso, \\ Av. São Sebastião, 2819, CEP 64202-020 Parnaíba -PI, Brasil \\ ${ }^{2}$ Universidade Estadual Paulista, Departamento de Ciências Biológicas, Faculdade de Ciências \\ Caixa Postal 473, CEP 17001-970 Bauru - SP, Brasil \\ ${ }^{3}$ Universidade Federal do Piauí, Curso de Medicina Veterinária, Campus "Prof. Cinobelina Elvas" \\ Br 135, km 03, CEP 64900-000, Bom Jesus -PI, Brasil \\ * Autor para correspondência \\ guilherme.ferreira@ufpi.edu.br
}

Submetido em 22/10/2014

Aceito para publicação em 31/03/2015

\section{Resumo}

O detalhamento da osteologia craniana de Milvago chimango e M. chimachima, contribui para assegurar a monofilia do gênero quando comparado a outras aves e a outros falconídeos. O estudo foi realizado com os crânios de três espécimes de Milvago chimango e três de Milvago chimachima, pertencentes a Divisão de Aves do Museu de História Natural Smithsonian Institution. A descrição craniana foi comparativa e ilustrada por fotos nas vistas: dorsal, ventral, caudal e lateral; sendo as espécies estudas comparados com Falconideos e algumas outras aves. Os caracteres exclusivos de M. chimango são: razão entre a distância da porção distal do osso lacrimal ao arco da órbita e deste até a arco jugal é de 1/5; ausência dos processos transpalatinos; ausência do processo dorsal do osso pterigoide; crânio mais robusto; osso paraoccipital mais desenvolvido laterolateralmente; osso lacrimal menor e mais rostral; osso palatino aproximadamente duas vezes mais largo; osso ectetmoide mais robusto e longo. Em M. chimachima são: presença de projeções na extremidade caudal do osso palatino; desenvolvido processo dorsal do osso pterigoide; fossa temporal mais afilada dorsoventralmente; crista nucal transversa delineada e visível; narina mais próxima à sutura frontonasal. A osteologia craniana forneceu caracteres diagnósticos de cada uma das espécies e do gênero, quando comparados a outras aves e aos falconídeos.

Palavras-chave: Anatomia; Crânio; Morfologia

\section{Abstract}

Principle features of the cranial osteology of Milvago chimango (Vieillot, 1816) and Milvago chimachima (Vieillot, 1816) (Aves: Falconidae). Knowledge of details of the cranial osteology of Milvago 
chimango and M. chimachima helps to ensure the monophyly of genus when compared to other birds and falcons. We conducted a study using skulls of three Milvago chimango and three Milvago chimachima specimens from the Smithsonian Institution National Museum of Natural History's Division of Birds. The cranial descriptions were comparative and illustrated by photos from the dorsal, ventral, and lateral flow perspectives. The studied species were then compared with falcons and other birds. Milvago chimango possesses several unique characters, including: the ratio of the distance from the distal portion of the lacrimal bone to the orbital arc, and from the orbital arc to the buccal arc is (1/5); absence of transpalate processes; lack of dorsal process on the pterygoid bone; more robust skull; paraoccipital bone more developed laterally; lacrimal bone smaller and more rostral; palatine bone approximately twice as wide; ethmoide bone more robust and long. In M. chimachima, the distinguishing features are: presence of projections at the caudal end of the palatine bone; developed dorsal process of the pterygoid bone; temporal fossa more tapered dorsoventrally; transverse nuchal crest outlined and visible; nostril closer to the frontonasal suture. Cranial osteology successfully provided diagnostic characters of each species and gender compared to other birds and falcons.

Key words: Anatomy; Morphology; Skull

\section{Introdução}

Os falconídeos constituem uma família de aves de rapina pertencentes à ordem Falconiformes composta por cerca de 60 espécies de aves, das quais 21 ocorrem no Brasil, distribuídas em oito gêneros (CBRO, 2011). Tal família é representada por aves como os falcões, o acauã, o carrapateiro e o caracará, caracterizando-se por constituir um grupo bastante heterogêneo apresentando diversidade morfológica e de massa corpórea assim como vocalização e formas de bicos diferenciadas.

Um comportamento pescador é descrito para duas espécies, o Milvago chimachima e o Daptrius ater, que usam técnicas diferentes para capturar este tipo de presa (MONTEIRO-FILHO, 1995; OLMOS; SAZIMA, 2009). Olmos e Sazima (2009) reportaram o Milvago chimango se alimentando de peixes pequenos em um estuário no Chile, na Costa Pacifica da América do Sul, um comportamento que parece não ter sido reportado e talvez não seja raro, ou uma tática restrita a esta espécie de falcão. A tática de "planar-pairar"' do M. chimango difere ligeiramente da tática de pesca adotada pelo pinhé (M. chimachima), espécie relacionada, que se lança sobre a presa a partir de um poleiro (MONTEIRO-FILHO, 1995; OLMOS; SAZIMA, 2009).

Trabalhos sobre a osteologia craniana dos Falconiformes são escassos na literatura. No que se refere à Milvago chimango, não foram encontrados trabalhos desta natureza. Estudos detalhados sobre as estruturas do crânio podem fornecer uma fonte importante de caracteres para análises filogenéticas. Segundo Zusi (1981) e Livezey e Zusi (2001), a diversidade morfológica é uma excelente fonte de caracteres utilizada para a construção de árvores filogenéticas, mas o crânio das aves poderia ser uma fonte ainda mais segura na análise cladística se novos caracteres e evidências anatômicas de homologias fossem encontradas.

Sendo assim, objetivou-se com esse trabalho descrever de forma detalhada e comparada a osteologia craniana de Milvago chimango e Milvago chimachima, identificando acidentes anatômicos cranianos de cada uma das espécies e do gênero, quando comparados a outras aves em especial a falconídeos.

\section{Material e Métodos}

Descreveu-se de forma detalhada a anatomia craniana de Milvago chimango e Milvago chimachima por meio da análise e comparação de peças osteológicas provenientes de Museus de História Natural. O estudo foi realizado com base na descrição dos ossos do crânio de três espécimes de Milvago chimango e três espécimes de Milvago chimachima, que fazem parte da coleção osteológica da Divisão de Aves do Museu de História Natural Smithsonian Institution (USNM), Washington, DC, EUA.

A descrição da osteologia craniana foi comparativa e ilustrada por fotos nas vistas dorsal, ventral, caudal e lateral, acompanhadas por legendas para facilitar a 
observação das estruturas, sendo que Milvago chimango e Milvago chimachima foram comparados com as seguintes espécies de Falconidae: Caracara plancus, Micrastur semitorquatus e Micrastur ruficollis.

Considerando que a maioria dos ossos cranianos é par e que existe simetria bilateral, a descrição osteológica se deu no singular. Para evitar subjetivismos buscou-se padronizar os termos utilizados na descrição anatômica, como "profundo", "desenvolvido", para estruturas osteológicas de difícil mensuração, como fossas ou cristas, sempre de maneira comparativa.

A nomenclatura para a descrição da osteologia e das demais estruturas associadas seguiu a Nomina Anatomica Avium (BAUMEL et al., 1993), e a classificação seguiu a apresentada pelo Comitê Brasileiro de Registros Ornitológicos (CBRO, 2011).

\section{Resultados e Discussão}

\section{Ossos do crânio (Ossa cranii)}

A região frontal de Milvago chimango (Osso frontal - Os frontale - F: Figuras 1 e 8) ocupa grande parte do teto craniano, cerca de metade do comprimento total da caixa craniana. A largura interorbital de $M$. chimango (1: Figura 1) é cerca de $3 / 7$ da região parietal, assim como pode ser observado em M. chimachima (1: Figura 8) e em Micrastur semitorquatus (SILVA et al., 2012). Ao passo que em Micrastur ruficollis (GUZZI et al., 2013) é de $2 / 5$. O estreitamento lateral do osso frontal na região das orbitas pode estar associado à ampliação do campo visual (BURTON, 1984), que aliada a posição frontal dos olhos (visão binocular), a proporção do tamanho do olho em relação ao crânio, a grande quantidade de células da retina e a presença de duas fóveas, podem auxiliar essas aves predadoras na obtenção de alimento. Porém, diferenças sutis com relação à largura interorbital dessas espécies podem aproximar as espécies do gênero Milvago de M. semitorquatus (SILVA et al., 2012), quando comparado as outras espécies de falconidae.

O osso frontal articula-se com o osso nasal (N: Figuras 1 e 8) rostralmente por meio da zona flexora craniofacial relativamente desenvolvida e parcialmente fundida medialmente, apresentando uma depressão do osso frontal nas espécies do gênero Milvago (DF: Figuras 1 e 8), tal como se observa em Micrastur semitorquatus (SILVA et al., 2012) e em Micrastur ruficollis (GUZZI et al., 2013). O compartilhamento desse caracter nos permite aproximar as espécies do gênero Milvago de M. semitorquatus (SILVA et al., 2012) e M. ruficollis (GUZZI et al., 2013). Em outros grupos de aves, como observado por Marceliano et al. (2007) em Geotrygon montana (Columbidae), o osso frontal apresenta a superfície dorsal plana, não possuindo qualquer depressão.

A parte ventral do osso lacrimal (L: Figuras 1, 2, 8 e 9) possui cerca de $1 / 3$ da largura da parte dorsal nas duas espécies do Milvago estudadas e em Micrastur semitorquatus (SILVA et al., 2012) e 1/2 em Micrastur ruficollis (GUZZI et al., 2013) e termina, nas três primeiras espécies, de forma arredondada, e em M. ruficollis (GUZZI et al., 2013), termina de forma afilada. A medida dessa distância aproxima Milvago de M. semitorquatus (SILVA et al., 2012) enquanto que $M$. ruficollis (GUZZI et al., 2013) exibem padrões diferentes das demais espécies. Segundo Cracraft (1968), em muitos grupos de aves o osso lacrimal pode estar fundido ao osso frontal e/ou ao osso nasal, e algumas vezes ao osso ectetmoide, em indivíduos adultos, sendo difícil determinar se este osso foi perdido ou encontra-se fundido a outro osso.

A razão entre a distância da parte ventral do osso lacrimal ao arco da órbita e deste até a arco jugal (AJ: Figuras 1, 2, 3, 8, 9 e 10) é de 1/5 em $M$. chimango, 1/4 em M. chimachima e Micrastur semitorquatus (SILVA et al., 2012) e de 1/7 em Micrastur ruficollis (GUZZI et al., 2013). Essa característica varia dentro e fora do gênero Milvago, não permitindo a sua polarização. A distância entre as partes ventrais dos dois ossos lacrimais atinge 3/4 da largura do osso parietal (2: Figuras 1 e 8) nas espécies de Milvago analisadas neste estudo e 12/13 em M. semitorquatus (SILVA et al., 2012) e M. ruficollis (GUZZI et al., 2013). Esse caracter aproxima as espécies de Milvago entre si e aproxima também as duas espécies de Micrastur entre si.

A ampla região parietal (Osso parietal-Os parietale - P: Figuras 1, 2, 8 e 9) limita-se lateroventralmente 
FIGURAS 1-7: Vistas do crânio e mandíbula de M. chimango. Figura 1 - Vista dorsal do crânio. Figura 2 - Vista lateral do crânio. Figura 3 - Vista ventral do crânio. Figura 4 - Vista caudal do crânio. Figura 5 - Vista dorsal da mandíbula. Figura 6 - Vista lateral da mandíbula. Figura 7 - Vista caudal dos ramos mandibulares. Escala quadriculado ao fundo 1x1 cm.

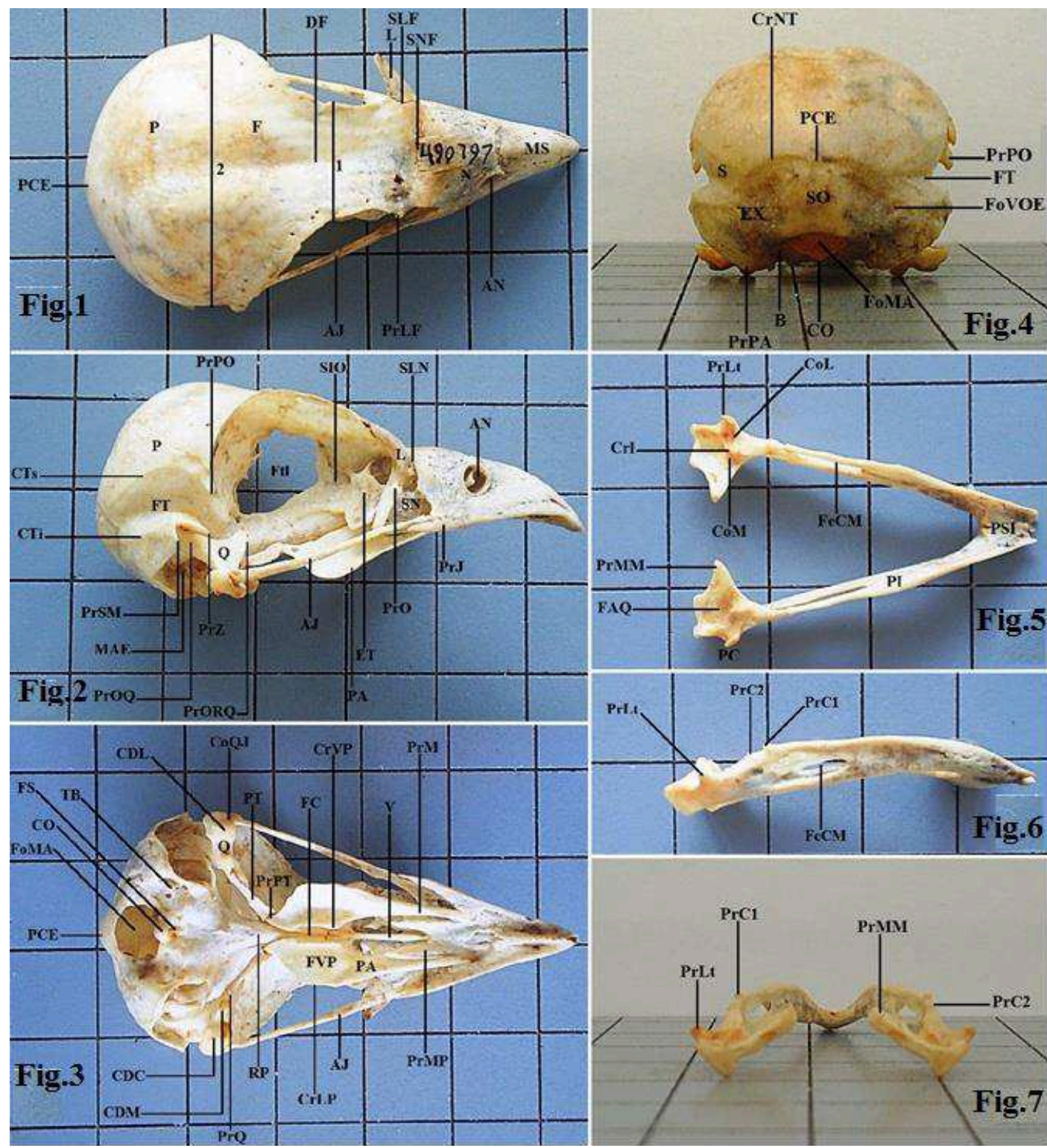

1: largura interorbital; 2: largura parietal; AJ: arco jugal; AN: abertura nasal; B: região basioccipital; CDC: côndilo caudal; CDL: côndilo lateral; CDM: côndilo medial; CO: côndilo occipital; CoL: cótila lateral; CoM: cótila medial; CoQJ: cótila quadratojugal; CrI: crista intercotilar; CrLP: crista lateral palatina; CrNT: crista nucal transversa; CrVP: crista ventral palatina; CTi: crista temporal inferior; CTs: crista temporal superior; DF: depressão frontal; ET: osso ectetmoide; EX: região exoccipital; F: osso frontal; FAQ: fossa articular quadrática; FC: fossa coanal; FeCM: fenestra caudal da mandíbula; FoMA: forâmen magno; FoVOE: forâmen da veia occipital externa; FS: fossa subcondilar; FT: fossa temporal; FtI: fontículo interorbital; FVP: fossa ventral palatina; L: osso lacrimal; MAE: meato acústico externo; MS: osso maxilar superior; N: osso nasal; P: osso parietal; PA: osso palatino; PCE: parte caudal; PCE: proeminência cerebelar; PI: parte intermediária; PrC1: processo coronoide1; PrC2: processo coronoide 2; PrJ: processo jugal; PrLF: processo lacrimal do osso frontal; PrLt: processo lateral da mandíbula; PrM: processo maxilar do osso palatino; PrMM: processo medial da mandíbula; PrMP: processo maxilopalatino do osso palatino; PrO: processo orbital do osso lacrimal; PrOQ: processo ótico do osso quadrado; PrORQ: processo orbital do osso quadrado; PrPA: processo paraoccipital do osso occipital; PrPO: processo pós-orbital do osso parietal; PrPT: processo pterigoide do osso palatino; PrQ: processo quadrático do osso pterigoide; PrSM: processo suprameático do osso temporal; PrZ: processo zigomático do osso temporal; PSI: parte sinfisial da mandíbula; PT: Osso pterigoide; Q: Osso quadrado; RP: rostro paraesfenoidal; S: Osso esquamosal; SFN: sutura frontonasal; SFP: sutura frontoparietal; SIO: septo interorbital; SLF: sutura lacrimofrontal; SLN: sutura lacrimonasal; SN: septo nasal; SO: supraoccipital; TB: tubérculo basilar; V: Osso vômer. 
FIGURAS 8-14: Vistas do crânio e mandíbula de M. chimachima. Figura 8 - Vista dorsal do crânio. Figura 9 - Vista lateral do crânio. Figura 10 - Vista ventral do crânio. Figura 11 - Vista caudal do crânio. Figura 12 - Vista dorsal da mandíbula. Figura 13 - Vista lateral da mandíbula. Figura 14 - Vista caudal dos ramos mandibulares. Escala quadriculado ao fundo 1x1 cm.
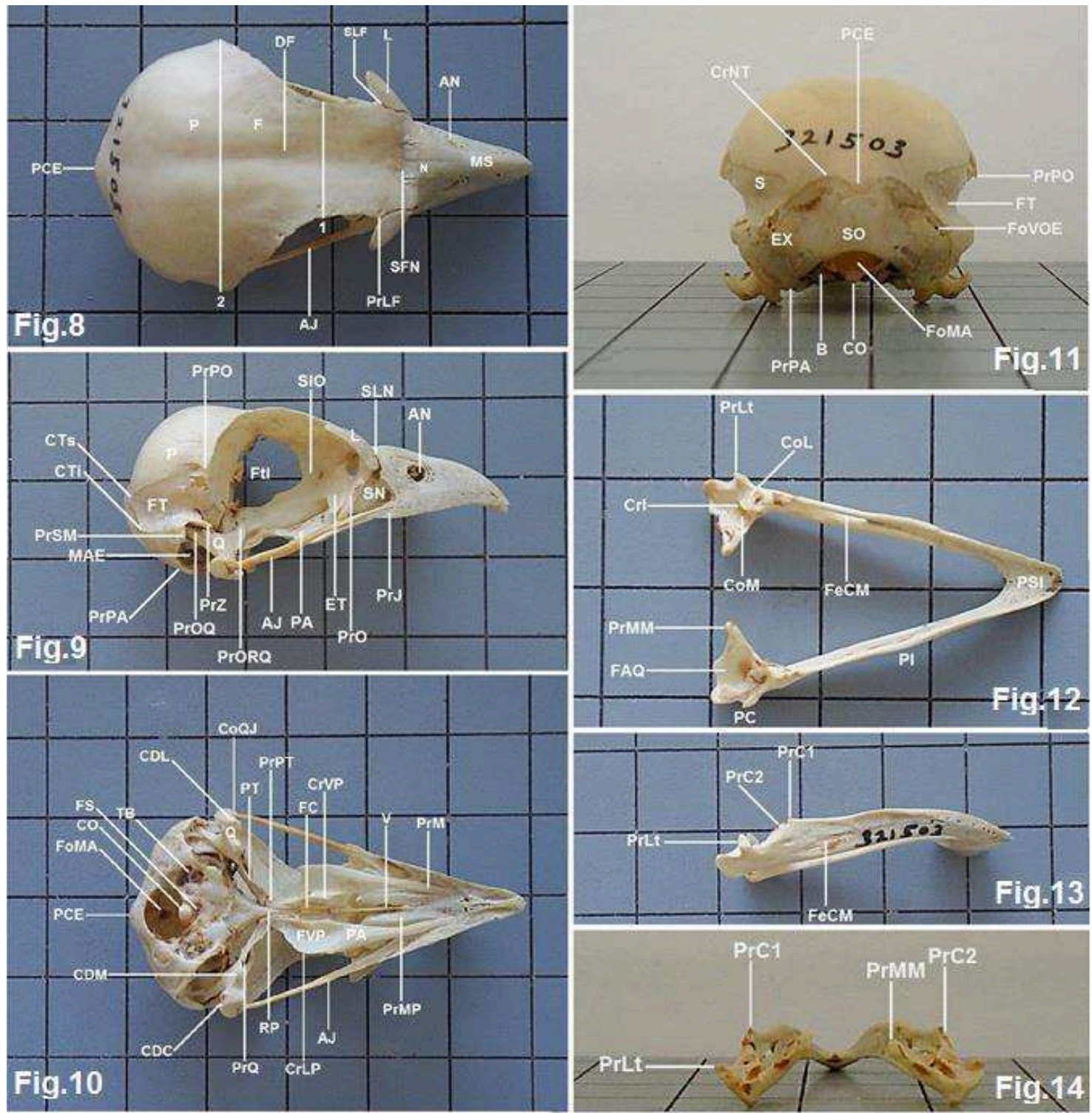

1: largura interorbital; 2: largura parietal; AN: abertura nasal; AJ: arco jugal; B: região basioccipital; CDC: côndilo caudal do osso quadrado; CDL: côndilo lateral do osso quadrado; CDM: côndilo medial do osso quadrado; CO: côndilo occipital do osso basoccipital; CoL: cótila lateral; CoM: cótila medial; CoQJ: cótila quadratojugal; CrI: crista intercotilar; CrLP: crista lateral palatina; CrNT: crista nucal transversa; CrVP: crista ventral palatina; CTi: crista temporal inferior; CTs: crista temporal superior; DF: depressão frontal; ET: osso ectetmoide; EX: região exoccipital; F: osso frontal; FAQ: fossa articular quadrática; FC: fossa coanal; FeCM: fenestra caudal da mandíbula; FoMA: forâmen magno; FoVOE: forâmen da veia occipital externa; FS: fossa subcondilar; FT: fossa temporal; FtI: fontículo interorbital; FVP: fossa ventral palatina; L: lacrimal; MAE: meato acústico externo; MS: maxila superior; N: osso nasal; P: osso parietal; PA: osso palatino; PC: parte caudal do ramo do osso mandibular; PCE: proeminência cerebelar; PI: parte intermediária do ramo do osso mandibular; PrC: processo coronoide; PrC1: processo coronoide1; PrC2: processo coronoide 2; PrJ: processo jugal; PrLF: processo lacrimal do osso frontal; PrLt: processo lateral da mandíbula; PrM: processo maxilar do osso palatino; PrMM: processo medial da mandíbula. PrMP: processo maxilopalatino; PrO: processo orbital do osso lacrimal; PrOQ: processo ótico do osso quadrado; PrORQ: processo orbital do quadrado; PrPA: processo paraoccipital do osso occipital; PrPO: processo pós-orbital do osso parietal; PrPT: processo pterigoide do osso palatino; PrQ: processo quadrático do osso pterigoide; PrSM: processo suprameático; PrZ: processo zigomático do osso temporal; PSI: parte sinfisial da mandíbula; PT: osso pterigoide; Q: osso quadrado; RP: rostro paraesfenoidal; S: esquamosal; SFN: sutura frontonasal; SIO: septo interorbital; SLF: sutura lacrimofrontal; SLN: sutura lacrimonasal; SN: septo nasal; SO: supraoccipital; TB: tubérculo basilar; V: osso vômer. 
com a região esquamosal por meio da conspícua crista temporal (Crista temporalis, CTs: Figuras 2 e 9); laterocaudalmente contata-se com a região occipital por meio da discreta crista nucal transversa (crista nuchalis transversa-CrNT: Figuras 4 e 11). A razão encontrada entre o diâmetro interorbital e a região parietal varia de 2,3 vezes para as duas espécies de Milvago descritas, em Micrastur semitorquatus (SILVA et al., 2012) é de 2,35 vezes; e em Micrastur ruficollis (GUZZI et al., 2013) é de 2,5 vezes. Esse caráter aproxima as duas espécies de Milvago.

A região esquamosal (Osso escamosal - Os squamosum - S: Figuras 4 e 11) é delimitada laterodorsalmente pela crista temporal (Crista temporalis,CTs: Figuras 2 e 9) que ocupa grande parte da porção lateral do crânio, caudal ao processo pósorbital; ventrocaudalmente pelo pronunciado processo suprameático observado em $M$. chimango (Proc. suprameaticus - PrSM: Figura 2), M. chimachima (PrSM: Figura 9) e Micrastur ruficollis (GUZZI et al., 2013), porém em Micrastur semitorquatus (SILVA et al., 2012) tal processo encontra-se pouco desenvolvido. A presença desse processo desenvolvido aproxima todas as espécies estudadas entre si, exceto em M. semitorquatus. O processo suprameático pouco desenvolvimento presente nas espécies de Milvago é observado em outras espécies de aves, como Crotophaginae (POSSO; DONATELLI, 2006). Já em $G$. montana (MARCELIANO et al., 2007), esse processo não foi observado, tampouco nos Coraciiformes (PASCOTTO et al., 2006), Trogonidae, Galbulidae, Capitonidae e Drendocolaptidae (DONATELLI, 1992).

A fossa temporal é, de forma geral, rasa, mas é mais profunda na parte proximal aos processos pósorbital e zigomático e mais rasa na parte ventral em $M$. chimango (Fossa temporalis - FT: Figuras 2 e 4), $M$. chimachima (FT: Figuras 9 e 11), Micrastur ruficollis (GUZZI et al., 2013) e Micrastur semitorquatus (SILVA et al., 2012), sendo delimitada dorsalmente pela crista temporal superior (CTs: Figuras 2 e 9) e ventralmente pela crista nucal transversa (CrNT: Figuras 4 e 11). Essa parece ser uma característica compartilhada por todas as espécies de falconídeos citados acima. Porém, em $M$. chimachima tal estrutura se apresenta mais profunda que nas demais. A profundidade da fossa temporal depende do desenvolvimento do sistema adutor mandibular externo, ou seja, quanto mais desenvolvido este sistema muscular maior a profundidade da fossa temporal (MORIOKA, 1974). Segundo Marceliano et al. (2007), em G. montana a fossa temporal é pequena, rasa e restrita à porção rostroventral da região esquamosal, ventrocaudalmente ao processo pós-orbital. Na região temporal, a família Nyctibiidae apresenta uma curta e profunda fossa temporal (COSTA; DONATELLI, 2009). De acordo com Pascotto et al. (2006), nos Coraciiformes podem ser encontrados três estágios de desenvolvimento da fossa temporal. O maior desenvolvimento (extensão e profundidade) é encontrado nos membros da Família Alcedinidae, sendo que neles as fossas temporais direita e esquerda encontram-se dorsalmente na linha mediana do crânio, salva raras exceções em que estão muito próximas. O menor desenvolvimento (extensão e profundidade) pode ser observado em Todidae, Leptosomidae, Upupidae e Phoeniculidae, sendo que nestes grupos a fossa temporal é praticamente plana. Já em Momotidae, Meropidae, Caraciidae, Bracypteraciidae e Bucerotidae a fossa temporal mostra desenvolvimento intermediário, sendo sua porção lateral mais profunda que sua porção dorsal, semelhante ao padrão encontrado nos Piciformes, segundo os mesmos autores. A fossa temporal profunda dorsalmente e rasa ventralmente é o local de surgimento das fibras do músculo adutor mandibular externo rostral temporal (M. adductor mandibulae externus rostralis temporalis) (DONATELLI; MARCELIANO, 2007). Essa diferença na profundidade da fossa temporal entre esses grupos pode estar relacionada a diferença na dieta, pois enquanto os falconídeos são, em sua maioria, carnívoros, os columbídeos são frugívoros e granívoros, e os Piciformes, em regra geral, são insetívoros. A fossa temporal profunda dos falconídeos indica que o músculo adutor mandibular é bem desenvolvido, auxiliando-o na caça de presas bem maiores que eles (ANTAS, 2005), podendo estar relacionado também à dilaceração das presas, durante o processo de alimentação.

A região esquamosal projeta-se rostralmente e forma o processo zigomático (Proc. zigomaticus - PrZ: Figuras 2 e 9). Tal processo apresenta-se longo e alargado em sua base e tem forma de um triângulo característico, 
levemente torcido sobre si mesmo, ocupando cerca de $45 \%$ da distância entre a sua origem e o arco jugal, ao passo que em Micrastur ruficollis (GUZZI et al., 2013) e Micrastur semitorquatus (SILVA et al., 2012) atinge $30 \%$. Essa característica aproxima claramente as espécies do gênero Milvago, com o maior processo zigomático, aproxima também as espécies estudadas de Micrastur, onde esse processo apresenta-se mais curto, ao passo que em $C$. plancus ela é intermediária. Com base no trabalho realizado por Marceliano et al. (2007) sobre a osteologia craniana de G. Montana verificou-se que o processo zigomático é pouco conspícuo. Migotto (2008) verificou três condições distintas para o processo zigomático - ausente ou vestigial (Elanus leucurus), curto (Buteo buteo) e desenvolvido (Pandion haliaetus).

No estudo realizado por Pascotto et al. (2006), o termo "processo zigomático" foi substituído pelo termo processo esquamosal como sugerido por Jollie (1957) e seguido por outros autores (HÖFLING; GASC, 1984; DONATELLI, 1992; 1996; POSSO; DONATELLI, 2006) pelo fato do termo zigomático estar associado ao arco zigomático dos mamíferos. Desta forma, constatouse, através do trabalho desenvolvido por Pascotto et al. (2006), que o processo esquamosal assume diferentes tamanhos nos Coraciiformes. Segundo Donatelli (1996), o processo esquamosal proporciona uma grande área de superfície para origem das aponeuroses dos músculos adutores mandibulares externos. O processo zigomático possui as faces dorsal, ventral, lateral e medial bem delimitadas, evidenciando os locais de origem dos músculos adutor mandibular externo rostral e medial (M. adductor mandibulae externus rostralis lateralis e medialis).

A região esquamosal delimita-se rostromedialmente com a região laterosfenoide e rostrodorsalmente com a região frontal por meio do processo pós-orbital (Proc. postorbitalis - PrPO: Figuras 2, 4, 9 e 11). Tal processo é curto e robusto, com base larga e levemente torcido sobre si mesmo nas espécies do gênero Milvago; já em Micrastur ruficollis (GUZZI et al., 2013) e Micrastur semitorquatus (SILVA et al., 2012) tal processo é longo e robusto, com base larga e levemente torcido sobre si mesmo. Esse caracter aproxima as espécies de Milvago, quando comparado às demais espécies estudadas.
A região occipital compreende os ossos supraoccipital (Os supraoccipitale - SO: Figuras 4 e 11), exoccipital (Os exoccipitale - EX: Figuras 4 e 11) e basioccipital (Os basioccipitale - B: Figuras 4 e 11) fusionados. Limita-se dorsolateralmente com a região esquamosal por meio do meato acústico externo (MAE: Figuras 2 e 9) e da crista nucal transversa.

A região do osso exoccipital delimita-se com a ala timpânica por meio do pronunciado processo paraoccipital (Proc. paraoccipitalis - PrPA: Figuras 4 e 11). O seu limite com a região basioccipital é marcado pela presença de forames por onde passam nervos (Forâme n. glossofaríngeo - Foramen $n$. glossopharingealis; n. vago - n.vagi e canal do n. hipoglosso - canalis n. hypoglossi) e de canais por onde passam vasos (Óstio do canal carotido - Ostium canalis carotici e Óstio do canal oftálmico externo ostium canalis ophthalmici externi) e pelo processo condilar, delimita-se rostralateralmente pelo osso esfenoide e dorsalmente pelo osso occipital e pelo forâme magno. A região do osso basioccipital abriga o conspícuo côndilo occipital (Condylus occipitalis - CO: Figuras 3, 4, $10 \mathrm{e}$ 11) e a profunda fossa subcondilar (Fossa subcondylaris - FS: Figuras 3 e 10); Migotto (2008) averiguou duas condições distintas para fossa subcondilar: rasa (Rosthramus sociabilis) ou profunda (Parabuteo unicinctus). A fossa subcondilar é circundada por dois tubérculos basilares bem desenvolvidos (Tuberculum basilare - TB: Figuras 3 e 10). Caudomedialmente observa-se o largo forâme magno (Foramen magno FoMA: Figuras 3, 4, 10, e 11). A proeminência cerebelar (Proeminentia cerebellaris) da região do osso supraoccipital é saliente e afilada em $M$. chimango (PCE: Figuras 1, 3 e 4), em M. chimachima (PCE: Figuras 8, 10 e 11) e em Micrastur ruficollis (GUZZI et al., 2013); diferindo-se da proeminência cerebelar (Proeminentia cerebellaris) pouco saliente observada em Micrastur semitorquatus (SILVA et al., 2012), e em outros grupos de aves, como em G. montana (MARCELIANO et al., 2007). No trabalho realizado por Migotto (2008) a proeminência cerebelar exibe duas condições distintas: pouco diferenciada (Busarellus nigricollis) e inflada (Heterospizias meridionalis). A região basioccipital (B: Figuras 4 e 11) continua-se rostralmente com a região basisfenoide por meio do tubérculo basilar (Tuberculum 
basilare - TB: Figuras 3 e 10). O osso basisfenoide (Os basisphenoidale) é alargada em sua base, mas se estreita rostralmente para se fundir com a região do osso paraesfenoide (Os parasphenoidale). O osso paraesfenoide é composta pela lamina paraesfenoidal (Lamina parasphenoidalis), pela asa paraesfenoidal (Ala parasphenoidalis) e pelos processos lateral e medial do osso paraesfenoide. Estes acidentes anatômicos localizam-se medialmente ao osso quadrado, quando todos somadas à região basioccipital formam a maior parte do assoalho craniano.

Da região do osso paraesfenoide ( $O S$ parasphenoidale), destaca-se a projeção rostro paraesfenoidal (Rostrum parasphenoidale - RP: Figuras 3 e 10) que é triangular e estreita-se medialmente formando uma lâmina que alcança $50 \%$ da distância do côndilo occipital e os pés pterigoide nas espécies do gênero Milvago e Micrastur ruficollis (GUZZI et al., 2013), enquanto que em Micrastur semitorquatus (SILVA et al., 2012) alcança apenas 40\% dessa distância, e em todas as espécies estudadas essa estrutura se articula rostralmente com o palatino (PA: Figuras 2, 3, 9 e 10) e rostrolateralmente com o pterigoide; $M$. semitorquatus (SILVA et al., 2012) apresenta um rostroparaesfenoide mais curto do que as demais espécies estudadas.

\section{Órbita (Orbita)}

O osso ectetmoide (Os ectethmoidale - ET: Figuras 2 e 9) apresenta-se fundido ao osso lacrimal em toda sua extensão, apresentando a forma da letra "D" deitada e um processo pronunciado nas espécies do gênero Milvago, Micrastur ruficollis (GUZZI et al., 2013) e Micrastur semitorquatus (SILVA et al. 2012). Projeta-se lateralmente e sua extremidade lateral fundese ao processo orbital do osso lacrimal (PrO: Figuras 2 e 9), formando o complexo lacrimal-ectetmoide (CRACRAFT, 1968). Quando comparado a outros grupos de aves, o osso ectetmoide pode apresentar variação, como o observado em G. montana (MARCELIANO et al., 2007) onde o osso ectetmoide funde-se ao septo interorbital medialmente e ao osso mesetmoide em sua porção rostromedial e dispõe-se como uma fina lâmina que abrange a porção rostral da órbita. Cracraft (1968) discutiu a função do complexo lacrimal-ectetmoide para as aves, relacionando o desenvolvimento de tais estruturas às pressões seletivas que atuam sobre tais processos, particularmente relacionando aos hábitos alimentares. Em sua face nasal, rostrolateralmente fundese com a face laterocaudal da região do osso frontal e do osso lacrimal. Entre esses dois ossos há uma pequena expansão lateral do osso frontal, denominada processo lacrimal do osso frontal no gênero Milvago (PrLF: Figuras 1 e 8), M. semitorquatus (SILVA et al., 2012) e M. ruficollis (GUZZI et al., 2013). Essa característica difere daquela encontrada por Marceliano et al. (2007), em G. montana (Columbidae), na qual não se observa a evidencia dessa sutura na região de fusão do osso frontal com o osso lacrimal. Rostromedialmente funde-se com a região rostral do septo interorbital.

O osso ectetmoide é bem desenvolvido e de fácil delimitação na órbita das duas espécies de Milvago, Micrastur ruficollis (GUZZI et al., 2013) e Micrastur semitorquatus (SILVA et al. 2012). Este é muito desenvolvido nos Nyctibiidae (COSTA; DONATELLI, 2009), apresentando uma forma globosa bastante característica, corroborando o descrito por Cracraft (1968).

\section{Ossos da face (Ossa faciei) Osso maxilar (Os maxillare)}

A maxila ou região maxilar (MS: - Figuras 1 e 8) é formada pela fusão dos ossos pré-maxilares (Os premaxillare), ossos maxilares (Os maxillare) e ossos nasais (Os nasale); tem cerca de $2 / 5$ do comprimento total do crânio no gênero Milvago e Micrastur semitorquatus (SILVA et al., 2012), ao passo que em Micrastur ruficollis (GUZZI et al., 2013) tem cerca de $1 / 3$ do comprimento total do crânio. Em Nyctibiidae (COSTA; DONATELLI, 2009) esta região corresponde a cerca de $40 \%$ do comprimento total do crânio em Nyctibiusgriseus, $N$. grandis, $N$. jamaicensis, N.aethereus e $N$. leucopterus e $45 \%$ em $N$. bracteatus. Em G. montana (MARCELIANO et al., 2007), a maxila não está disposta em um mesmo plano que o crânio, orienta-se ventralmente, com um ângulo de aproximadamente $40^{\circ}$. É longo e estreito, atingindo cerca de 50\% do comprimento total do crânio. Segundo Zusi (1984), todas as aves modernas têm a capacidade 
de mover o osso maxilar ou uma parte dele em relação à cabeça. Essa propriedade funcional é conhecida como cinese craniana, e seu mecanismo inclui, além do osso maxilar, várias outras partes do crânio, o osso da mandíbula, o osso quadrado, o osso palatino, os arcos jugais, certos músculos da mandíbula, ligamentos e articulações diversas. Assim, muito da morfologia do crânio das aves refere-se à cinese craniana.

As narinas (AN: - Figuras 1, 2, 8 e 9) têm um formato circular e caracterizam-se como do tipo holorrina em todas as espécies estudadas, assim como em Nyctibiidae (COSTA; DONATELLI, 2009), condição encontrada nas aves com crânio procinético (BOCK, 1964; 1966; ZUSI, 1984). Em G. Montana (MARCELIANO et al., 2007), a abertura nasal externa é ampla e estreita, abrangendo a maior parte da face dorsal do osso maxilar e não há septo nasal evidente separando as duas aberturas nasais. Já nos Coraciiformes (PASCOTTO et al., 2006), é ampla e oval, ocupando $40 \%$ do comprimento do osso maxilar em Todidae e Brachypteraciidae; é reduzida em Coraciidae, Cerylinae e Alcedininae, ocupando cerca de $20 \%$ do comprimento do osso maxilar, e cerca de 15\% em Leptosomidae; é extremamente reduzida em Phoeniculidae, Upupidae e Bucerotidae, nos quais ocupando cerca de $5 \%$ do comprimento do osso maxilar. Migotto (2008) verificou duas condições diferentes para o septo nasal- não perfurado (ou com pequeno forâme) (Haliaeetus leucocephalus) e perfurado (Aquila chrysaetos).

\section{Palato (Palatum)}

A região do palato é formada pelos ossos palatinos, pterigoides e vômer, sendo caracterizado como do tipo esquizognato. Pois, segundo Bellairs e Jenkin (1960), o palato é esquizognato quando os processos maxilopalatinos não se tocam medialmente deixando uma fenda entre os ossos onde o osso vômer se localiza. Esse mesmo tipo de palato foi observado por Costa e Donatelli (2009) nos Nyctibiidae. Segundo Pascotto et al. (2006), o palato dos Coraciiformes é desmognato (exceto em Todidae), caracterizado basicamente pela completa fusão mediana dos maxilopalatinos, diretamente ou pela intermediação do septo nasal. O processo maxilar do osso palatino (Proc. maxillaris - PrM: Figuras 3 e 10) conecta a lamela caudolateral palatina à base do osso maxilar. Este processo é longo e estreito em sua extensão em todas as espécies. A lâmina caudolateral palatina (Lamella caudolateralis) da porção lateral é delimitada medialmente pela proeminente crista ventral palatina (Crista ventralis - CrVP: Figuras 3 e 10) da porção coanal, pelo processo transpalatino e lateralmente pela curta crista lateral palatina (Crista lateralis-CrLP: Figuras 3 e 10); sendo pouco conspícua em G. montana (MARCELIANO et al., 2007). No trabalho desenvolvido por Migotto (2008) a crista lateral dos ossos palatinos foi analisada sob três condições diferentes: plana (Elanus leucurus), inclinada lateralmente (Harpyhaliaetus coronatus) e inclinada ventralmente (Gyps fulvus); Entre as cristas lateral e ventral do osso palatino observa-se uma profunda fossa ventral palatina (Fossa ventralis FVP: Figuras 3 e 10). Assim, a fossa ventral palatina é curta, estreita, profunda e forma a maior parte da lamela caudolateral palatina em M. chimango; enquanto que em M. chimachima, Micrastur semitorquatus (SILVA et al., 2012) e Micrastur ruficollis (GUZZI et al., 2013) é extensa, larga, profunda e forma a maior parte da lamela caudolateral palatina.

As cristas ventrais palatinas são curtas, proeminentes e encontram-se na região de articulação com o osso paraesfenoide em ambas as espécies de Milvago, ao passo que nas demais espécies estudadas são longas. A articulação com o osso pterigoide faz-se dorsolaterocaudalmente, por meio da porção pterigoide palatina que apresenta um curto processo pterigoide do palatino (PrPT: Figuras 3 e 10). As cristas mediais palatinas (Crista medialis) são proeminentes, terminando de forma afilada, situando-se mais medialmente e delimitam a fossa coanal (FC: Figuras 3 e 10). A porção coanal é estreita e mais desenvolvida se comparada à porção lateral descrita acima. O processo transpalatino está ausente em Milvago Chimango, M. chimachima e Micrastur semitorquatus (SILVA et al., 2012), porém está presente em Micrastur ruficollis (GUZZI et al., 2013). Nos Coraciiformes (PASCOTTO et al., 2006) tal processo está presente em Alcedininae, Cerylinae, Daceloninae, Phaeniculidae e Upupidae, estando ausente nas demais famílias.

O osso vômer (V: Figuras 3 e 10) apresentase como uma lâmina longa e estreita, achatada 
laterolateralmente, pontiagudo rostralmente; articula-se caudalmente com a porção coanal do palatino e com o rostro paraesfenoide (RP: Figuras 3 e 10) por meio da face articular vomeral em $M$. chimango, $M$. chimachima, Micrastur ruficollis (GUZZI et al., 2013) e Micrastur semitorquatus (SILVA et al., 2012); tal como em G. montana (MARCELIANO et al., 2007). Dessa forma, todos os falconídeos aqui analisados compartilham a mesma característica. Migotto (2008) analisou o osso vômer sob duas perspectivas: o carácter apresenta duas condições - ausente (Buteo buteo) ou presente (Rosthramus sociabilis) e o outro caractere quanto a sua extensão, podendo atingir a porção rostral dos processos maxilopalatinos do osso maxilar (Buteo buteo) ou não atingindo (Rosthramus sociabilis). Segundo Costa e Donatelli (2009), o osso vômer apresenta estrutura bastante variável entre as espécies de Nyctibiidae. Em $M$. semitorquatus (SILVA et al., 2012), o osso vômer é bem desenvolvido e laminar (achatado lateralmente). Alguns autores (p. ex. ZUSI, 1993) consideram o osso vômer uma estrutura importante do ponto de vista sistemático, e as variações observadas nesse osso dentro da família, a exemplo de diversas outras características do crânio, se mostram bastantes informativas para uma futura análise filogenética da família. O osso vômer dos Coraciiformes (PASCOTTO et al., 2006) é ausente ou rudimentar, quando presente este é extremamente delgado e com a extremidade distal afilada.

O osso quadrado localiza-se em uma região central do crânio e interliga o palato, a caixa craniana e a mandíbula, desempenhando um papel fundamental na cinética craniana. Apresenta um corpo quadrático (corpus ossi quadrati-Q: Figuras 2, 3, 9 e 10) central de onde partem três processos: orbital, ótico e mandibular. Embora ainda haja discordâncias sobre os movimentos exatos do osso quadrado durante a abertura do bico, praticamente todos os autores concordam no que diz respeito à função desempenhada pelo quadrado durante a cinese craniana: ele é o ponto inicial a partir do qual se originam todos os movimentos cranianos (BOCK, 1964; BÜHLER, 1981; ZUSI, 1981; BURTON, 1984). A articulação da mandíbula com a caixa craniana é realizada nas aves principalmente por meio do osso quadrado (ZUSI, 1984; PROCTOR; LINCH, 1993; KARDONG, 1998), originando a força que será transmitida pelos ossos pterigoide e palatino ao osso maxilar.

O processo orbital do osso quadrado (Proc. orbitalis - PrORQ: Figuras 2 e 9) projeta-se rostromedialmente na órbita; apresenta-se curto e largo, afilando-se tenuamente ventralmente e terminando na forma de bulbo. Assume claramente uma posição oblíqua na órbita. Em Nyctibiidae (COSTA; DONATELLI, 2009), o osso quadrado apresenta-se alongado, verticalmente posicionado, e com um processo orbital extremamente reduzido, quase vestigial. Tal fato é também observado por Cracraft (1981) e Mayr (2002), sendo uma condição bastante distinta de outros grupos de aves. Segundo Bock (1964) e Bühler (1981), esse processo é o local de origem do músculo pseudotemporal profundo (m. pseudotemporalis profundus) e importante região de inserção de músculos relacionados a movimentos de elevação da mandíbula. No entanto, o fato do processo orbital do osso quadrado ser reduzido não significa necessariamente que alguns músculos estejam ausentes, como afirmam Pascotto et al. (2006) para outros grupos de aves.

O processo ótico do osso quadrado (Proc. oticus - PrOQ: Figuras 2 e 9) é longo e alargado a partir do corpo quadrático. Este processo em G. montana de acordo com Marceliano et al. (2007) é similar ao de $C$. livia (GENNIP, 1986) mostrando-se bem desenvolvido, e difere daquele de C. squammata (ANDRELA; DONATELLI, 1995). Já o processo orbital do quadrado possui morfologia distinta nessas três espécies, sendo mais desenvolvido em C. livia. Articula-se com a caixa craniana em dois locais distintos: 1) por meio do processo ótico externo do osso quadrado (Capitulum squamosum), apoiado na face ventrocaudal do processo zigomático; e 2) por meio do processo ótico interno do osso quadrado (Capitulum oticum) que se articula com a cótila quadrática ótica (cotylae quadraticae otici) dos ossos proótico e opistótico.

O processo mandibular do osso quadrado apresenta quatro côndilos distintos: o côndilo medial (Condylus medialis - CDM: Figuras 3 e 10) que é arredondado; Migotto (2008) analisou a forma do côndilo medial do osso quadrado, atribuindo a ele duas condições díspares: arredondada (Buteo buteo) ou alongada (Rosthramus sociabilis); o côndilo lateral (Condylus 
lateralis - CDL: Figuras 3 e 10) que é longo e mais robusto que o medial, articulando-se com o arco jugal lateralmente a partir de um processo dorsal; o côndilo caudal (Condylus caudalis - CDC: Figuras 3 e 10) é uma extensão do côndilo lateral, sendo fundido ele como se constata em $M$. chimango e $M$. chimachima. A condição apresentada aproxima C. plancus das espécies do gênero Milvago. No estudo desenvolvido por Migotto (2008) o côndilo caudal do osso quadrado foi analisado sob duas condições distintas; saliente (Buteo buteo) ou pouco desenvolvido (Rostrhamus sociabilis). Nos Coraciiformes (PASCOTTO et al., 2006), o côndilo caudal do processo mandibular do osso quadrado encontra-se fundido lateroventralmente com o côndilo lateral em Phoeniculidae, Upupidae e Leptosomidae, sendo distintos nos demais grupos. Em Cerylinae ocorre variação especifica (PASCOTTO et al., 2006). E o côndilo do pterigoide, localizado medialmente, articula-se com o osso pterigoide por meio de sua face articular quadrática.

O osso pterigoide (Os pterygoideum - PT: Figuras 3 e 10) tem uma expansão rostral que se alarga rostrodorsalmente; articula-se com o osso palatino dorsolaterocaudalmente por meio de uma estrutura denominada pé pterigoide (pes pterygoidei). De uma forma geral, o osso pterigoide apresenta-se como uma lâmina relativamente achatada e disposta obliquamente na órbita. O osso pterigoide de G. montana (MARCELIANO et al., 2007) distingue-se daquele de C. squammata (ANDRELA; DONATELLI, 1995) por ser estreito e curvado; nesta última, o mesmo é curto e largo, sem evidências de curvatura. Nos Coraciiformes (PASCOTTO et al., 2006), o osso pterigoide é reto, exceto em Todidae, e em algumas espécies de Alcedininae. $\mathrm{O}$ osso pterigoide localiza-se entre os ossos quadrado e palatino e desempenha importante papel no aparato mandibular ao transmitir o movimento do osso quadrado ao osso palatino e vice-versa (GENNIP, 1986). Articulase laterocaudalmente com o osso quadrado por meio da face articular quadrática do processo quadrático do osso pterigoide (Proc. quadraticus - PrQ: Figuras 3 e 10).

$\mathrm{O}$ arco jugal apresenta o côndilo quadrático (Condylus quadraticus) que se articula com a cótila quadratojugal (Cotyla quadratojugalis-CoQJ: Figuras
3 e 10). O arco jugal em Nyctibiidae apresenta-se bastante arqueado lateralmente ao crânio, assim como observaram Baumel e Witmer (1993). O arco jugal exerce importante função na transmissão de forças do osso quadrado à região caudoventral do osso maxilar (BÜHLER, 1981).

\section{Osso da mandibula (Ossa mandibulae)}

A porção sinfisal da mandibula (PSI: Figuras 5 e 12) ocupa cerca de $1 / 6$ do comprimento total do osso da mandíbula nas duas espécies de Milvago, 1/5 em Micrastur semitorquatus (SILVA et al., 2012) e Micrastur ruficollis (GUZZI et al., 2013). Esse caracter apresenta-se de forma homogêneas nas espécies, mas separa claramente os gêneros. Em G. montana (MARCELIANO et al., 2007) reúne os dois ramos mandibulares. Esta parte é curta, atingindo somente cerca de $15 \%$ do comprimento total da mandíbula. Os ramos mandibulares são estreitos em sua porção rostral e alargam-se em sua porção medial e caudal. A porção intermédia (Pars intermedia - PI: Figuras 5 e 12) possui uma fenestra mandibular bem desenvolvida, mas rasa em toda a sua extensão, e a porção caudal (PC: Figuras 5 e 12) abriga a musculatura que movimenta os ossos maxilares, além de ser a área de articulação com o osso quadrado.

Na região dorsal da mandíbula, observa-se o bem desenvolvido processo coronoide (Proc. coronoideus - PrC1: Figuras 6, 7, 13 e 14) região do osso supraangular (Os supra-angulare), na altura da porção caudal da fenestra mandibular (FeCM: Figuras 6 e 13) presente nas duas espécies de Milvago, Micrastur semitorquatus (SILVA et al., 2012) e Micrastur ruficollis (GUZZI et al., 2013). Todas as espécies de falconídeos aqui analisados demonstram compartilhar essa mesma condição. De acordo com Marceliano et al. (2007), em seu estudo com G. montana (Columbidae) há o que se denominou processo pseudocoronoide do osso mandibular que é pouco conspícuo e localiza-se na porção rostral da cótila lateral. Baumel e Witmer (1993) consideram o processo dorsal da mandíbula como processo coronoide, mas alertam que o osso coronoide não participa na formação da mandíbula em alguns grupos de aves. Nos Coraciiformes (PASCOTTO et al., 2006) o processo coronoide 1 e o 
processo coronoide 2 são reduzidos, mas distintos entre si, exceto em Bucerotidae em que se apresentam fundidos. Em espécies do gênero Milvago dorsorostralmente, destaca-se o processo coronoide 2 (PrC2: Figuras 6, 7, 13 e 14), que é mais desenvolvido entre eles.

$\mathrm{Na}$ região dorsocaudal da mandíbula, pode ser observada a crista intercotilar (CrI: Figuras 5 e 12), pronunciada em Milvago. A cótila lateral (Cotyla lateralis - CoL: Figuras 5 e 12) e a cótila medial (Cotyla medialis - CoM: Figuras 5 e 12) mostramse conspícuas. O bem desenvolvido processo medial da mandíbula (Proc. medialis mandibulae - PrMM: Figuras 5, 7, 12 e 14) projeta-se dorsomedialmente, sendo largo em sua base afilando-se dorsalmente. O desenvolvimento do processo medial pode contribuir para evitar a desarticulação da mandíbula quando em movimento (BOCK, 1960).

$\mathrm{Na}$ mandíbula, está presente o bem desenvolvido processo lateral da mandíbula (PrLt: Figuras 5, 7, $12 \mathrm{e}$ 14), disposto caudalmente na sua parte caudal. Migotto (2008) analisou o processo lateral da mandíbula dos gaviões buteonineos e atribuiu a ele as condições: curto (Leptodon cayannensis) ou saliente (Parabuteo unicinctus). O tubérculo pseudotemporal da região medial da mandíbula (Tuberculum pseudotemporale) é inconspícuo no gênero Milvago, o que não difere muito do encontrado em G. montana (MARCELIANO et al., 2007) em que o tubérculo pseudotemporal é pouco conspícuo. Dentre os falconídeos aqui considerados apenas as duas espécies de Milvago apresentaram tal estrutura.

$\mathrm{Na}$ região caudal, destaca-se a fossa caudal (Fossa caudalis), bem desenvolvida, sendo rasa e larga; delimita-se lateralmente com a crista lateral, medialmente com a crista medial e dorsalmente com a crista transversa da fossa caudal. O processo retroarticular (Proc. retroarticularis) não foi observado nas duas espécies de Milvago e nem em Micrastur ruficollis (GUZZI et al., 2013), porém em Micrastur semitorquatus (SILVA et al., 2012) é reduzido, sendo de difícil visualização; Considerando a presença ou ausência do processo retroarticular, é possível aproximar as duas espécies de Milvago de M. ruficollis (GUZZI et al., 2013) separando-os de M. semitorquatus (SILVA et al., 2012). Em M. momota (PASCOTTO et al., 2006) é relativamente pronunciado. Migotto (2008) verificou a presença do processo retroarticular do ramo mandibular em gaviões buteonineos, e conferiu a este caractere três condições distintas: direcionado lateralmente (Harpia harpia), direcionado caudalmente (Parabuteo unicinctus) ou direcionado medialmente (Geranoaetus melanoleucus). Nos Coraciiformes (PASCOTTO et al., 2006) o típico processo retroarticular da mandíbula está presente apenas em Phoeniculidae, Upupidae e Bucerotidae. Segundo Marceliano et al. (2007), em seu estudo sobre G. montana (Columbiformes: Columbidae), apesar de diversas semelhanças nas estruturas cranianas dos Columbidae terem sido apontadas anteriormente, inúmeras diferenças foram constatadas entre os gêneros analisados, principalmente relativos ao grau de desenvolvimento; todavia, há estruturas que se apresentam em alguns gêneros, como o processo retroarticular do ramo mandibular e a projeção caudal palatina, as quais distinguem os gêneros de maneira mais efetiva que o simples desenvolvimento.

A descrição da osteologia craniana de $M$. chimango e $M$. chimachima fornece alguns caracteres comuns ao gênero Milvago. Dentre as características osteológicas analisadas e considerando as espécies utilizadas, aquelas que são exclusivas para $M$. chimachima são: projeções nas extremidades caudais do osso palatino; processo dorsal do osso pterigoide desenvolvido; fossa temporal termina de forma mais afilada dorsoventralmente; a crista nucal transversa mais delineada e visível e narina mais próxima à sutura frontonasal. Para $M$. chimango, são a razão entre a distância da parte distal do osso lacrimal ao arco da órbita e a razão do arco da órbita até a arco jugal que é de 1/5; a ausência de projeções nas extremidades caudais do osso palatino; e a ausência de um processo dorsal do osso pterigoide; crânio é mais robusto; osso paraoccipital mais desenvolvido laterolateralmente; osso lacrimal menor e posicionado mais rostral; osso palatino cerca de duas vezes mais largo e o osso ectetimoide mais robusto e longo.

Os caracteres que reúnem todos os falconídeos aqui analisados são: a presença de um processo lacrimal do osso frontal; a presença de osso ectetmoide bem desenvolvido; um osso vômer afilado e a presença de um processo coronoide do ramo mandibular. 


\section{Referências}

ANDRELA, S.; DONATELLI, R. J. Osteologia e miologia cranianas de Columbina squammata (Lesson, 1831) (Aves: Columbiformes). Naturalia, Rio Claro, v. 20, p. 107-123, 1995.

ANTAS, P. T. Z. Aves do Pantanal. RPPN. Sesc. 2005.

BAUMEL, J.; KING, A. S.; LUCAS, A. M.; BREAZILE, J. E.; EVANS, H. E. Nomina anatomica avium. London: Academic Press, 1993. $637 \mathrm{p}$.

BAUMEL, J.; WITMER, L. M. Osteology. In: BAUMEL, J.; KING, A. S.; BREAZILE, J. E.; EVANS, H. E.; VANDER BERGE, J. C. (Ed.). Handbook of Avian Anatomy: Nomina Anatomica Avium. 2 ed. Cambridge: Nut all Ornithologists Club, 1993. p. 45-132.

BELLAIRS, A. A.; JENKIN, C. R. The skeleton of birds. In: MARSHALL, J. A. (Ed.). Biology and Comparative Physiology of Birds. New York: Academic Press, 1960. p. 241-300.

BOCK, W. J. Secondary articulation of the avian mandible. Auk, Washington, v. 77, n. 1, p. 19- 55, 1960.

BOCK, W. J. Kinetics of the avian skull. Journal of the Morphology, Philadelphia, v. 114, p. 1-41, 1964.

BOCK, W. J. An approach to the functional analysis of the bill shape. Auk, Washington, v. 83, p. 10-51, 1966.

BÜHLER, P. Functional anatomy of the avian jaw apparatus, In: KING, A. S.; MCLELLAND, J. (Ed.). Form and functions in birds. Vol. 2. London: Academic Press, 1981. p. 439-468.

BURTON, P. J. K. Anatomy and evolution of the feeding apparatus in the avian orders Coraciiformes and Piciformes. Bulletin of the British Museum (Natural History), London, v. 47, n. 6, p. 331443, 1984.

CBRO - COMITÊ BRASILEIRO DE REGISTROS ORNITOLÓGICOS. Lista de aves do Brasil - 2011. 10 ed. Disponível em: <http://www.cbro.org.br>. Acesso em: 2 mar. 2012. COSTA, T. V. V.; DONATELLI, R. J. Osteologia craniana de Nyctibiidae (Aves, Caprimulgiformes). Papéis Avulsos de Zoologia, São Paulo, v. 49, p. 257-275, 2009.

CRACRAFT, J. The lacrimal-ectehmoid bone complex in bird: a single character analysis. American Midland Naturalist, Notre Dame, v. 80, n. 2, p. 316-359, 1968.

CRACRAFT, J. Toward a philogenetic classification of the recent birds of the world (class Aves). Auk, Washington, v. 98, n. 4, p. 681-714, 1981.

DONATELLI, R. J. Cranial osteology and miology of the jaw apparatus in the Galbulidae (Aves, Piciformes). Arquivos de Zoologia, São Paulo, v. 32, n. 1, p. 32, 1992.

DONATELLI, R. J. The jaw apparatus of the Neotropical and of the Afrotropical woodpeckers (Aves, Piciformes). Arquivos de Zoologia, São Paulo, v. 32, n. 3, p. 1-70, 1996.

DONATELLI, R. J.; MARCELIANO, M. L. V. Osteologia e miologia cranianas de Megaxenops parnaguae (Furnariidae: Philydorinae). Boletim do Museu Paraense Emílio Goeldi. Ciências Naturais, Belém, v. 2, n. 1, p. 183-215, 2007.

GENNIP, E. M. S. J. van. The osteology, arthrology and myology of the jaw apparatus on the pigeon (Patagioenas livia L.). Netherlands Journal of Zoology, Wageningen, v. 36, n. 1, p. 1-46, 1986.

GUZZI, A.; SANTOS, F. C. V.; FERREIRA, G. J.; DONATELLI, R. J. Comparative analysis of the cranial osteology and evolutionary traits of Micrastur gilvicollis, Micrastur ruficollis and Micrastur semitorquatus (Aves: Falconidae). Revista Nordestina de Zoologia, Recife, v. 7, p. 11-39, 2013.

HÖFLING, E.; GASC, J. P. Biomecanique du crane et du bec chez Ramphastos (Ramphastidae, Aves). Description des elements anatomiques. Gegenbaurs Morphologisches Jahrbuch, Heidelberg, v. 130, n. 1, p. 125-147, 1984.

JOLLIE, M. The head skeleton of the chicken and remarks on the anatomy of this region in other birds. Journal of Morphology, Philadelphia, v. 100, n. 4, p. 389-436, 1957.

KARDONG, K. V. Vertebrates: comparative anatomy, function, evolution. New York: McGraw-Hill, 1998. 747 p.

LIVEZEY, B. C.; ZUSI, R. L. Higher-order phylogenetics of modern Aves based on Comparative Anatomy. Netherlands Journal of Zoology, Wageningen, v. 51, n. 2, p. 179-205, 2001.

MARCELIANO, M. L. V.; DONATELLI, R. J.; POSSO, S. R. Osteologia craniana de Geotrygon montana (Linnaeus, 1758) (Columbiformes: Columbidae) comparada com os Columbiformes do Novo Mundo. Boletim do Museu Paraense Emílio Goeldi, Belém, v. 2, n. 3, p. 21-31, 2007.

MAYR, G. Osteological evidence for paraphyly of the avian order Caprimulgiformes (nightjars and allies). Journal of Ornithology, Heidelberg, v. 143, p. 82-97, 2002.

MIGOTTO, R. Inferência filogenética em gaviões buteoninos (Aves: Accipitridae), com base em caracteres osteológicos cranianos. 2008. 99 f. Dissertação (Mestrado em Ciências - Área Zoologia) - Universidade de São Paulo, São Paulo. 2008.

MONTEIRO-FILHO, E. L. A. Fishing behavior of yellow-headed caracara, Milvago chimachima (Falconidae) in southeast Brazil. Ciencia e Cultura, São Paulo, v. 47, n. 1/2, p. 86-87, 1995.

MORIOKA, H. Jaw musculature of swifts (Aves, Apodidae). Bulletin of the National Museum of Natural Science, Taichung, v. 17, n. 1, p. 1-16, 1974.

OLMOS, F.; SAZIMA, I. Fishing behaviour by Black Caracaras (Daptrius ater) in the Amazon. Biota Neotropica, Campinas, v. 9, n. 3, p. 399-401, 2009.

PASCOTTO, M. C.; HÖFLING, E.; DONATELLI, R. J. Osteologia craniana de Coraciiformes (Aves). Revista Brasileira de Zoologia, Curitiba, v. 23, n. 3, p. 841-864, 2006.

POSSO, S. R.; DONATELLI, R. J. Análise filogenética e implicações sistemáticas e evolutivas nos Cuculiforormes (Aves) com base na osteologia, comportamento e ecologia. Revista Brasileira de Zoologia, Curitiba, v. 23, n. 3, p. 608-629, 2006.

PROCTOR, N. S.; LINCH, P. J. Manual of ornithology: avian structure and function. New Haven: Yale University Press, 1993. $340 \mathrm{p}$.

SILVA, A. G.; FERREIRA, G. J.; DONATELLI, R. J.; GUZZI, A. Osteologia craniana de Micrastur semitorquatus (Vieillot, 1817) (Falconiformes: Falconidae). Comunicata Scientiae, Bom Jesus, v. 3, n. 1, p. 64-71, 2012.

ZUSI, R .L. Patterns of diversity in the avian skull. In: KING, A. S.; MCLELlAND, J. (Ed.). Form e function in birds. Vol. 1. London: Academic Press, 1981. p. 391-437.

ZUSI, R. L. A functional and evolutionary analysis of rhynchokinesisin birds. Smithsonian ContributionS to Zoology, Washington, v. 395, p. 1-40, 1984.

ZUSI, R. L. Patterns of diversity in the avian skull. In: HAKEN, J.; HALL, B. K. (Ed.). The skull. Vol. 2. Chicago: Chicago University Press, 1993. p. 391-437. 